\title{
Mercury Data from Small Lakes in Voyageurs National Park, Northern Minnesota, 2000-02
}

By Robert M. Goldstein, Mark E. Brigham, Luke Steuwe, and Michael A. Menheer

Open-File Report 03-480

Prepared in cooperation with the National Park Service and the Minnesota Pollution Control Agency 


\title{
U.S. DEPARTMENT OF THE INTERIOR
}

\section{Gale A. Norton, Secretary}

\section{U.S. GEOLOGICAL SURVEY}

\author{
Charles G. Groat, Director
}

Use of firm, trade, and brand names in this report is for identification purposes only and does not constitute endorsement by the U.S. Geological Survey.

Mounds View, Minnesota, 2003

For additional information write to:

U.S. Geological Survey

District Chief

2280 Woodale Drive

Mounds View, MN 55112

Copies of this report can be purchased from:

\section{U.S. Geological Survey}

Branch of Information Services

Box 25286, MS 517

Federal Center

Denver, CO 80225

Information regarding the USGS is available on the Internet via the World Wide Web.

You may connect to the USGS Home Page using the Universal Resource Locator (URL) at http://wwwrvares.er.usgs.gov.

You may also connect to the Minnesota District Home Page at http://mn.water.usgs.gov/.

For more information on all USGS reports and products (including maps, images, and computerized data), call 1-888-ASK-USGS

Open-File Report 03-480 


\section{CONTENTS}

Abstract

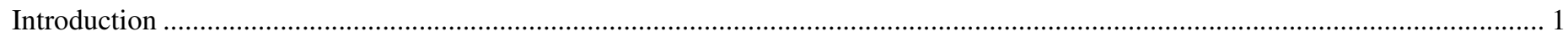

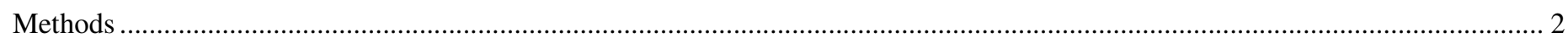

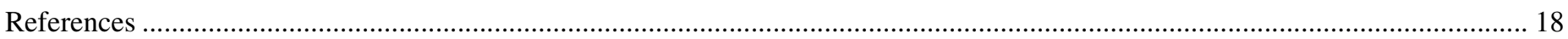

\section{ILLUSTRATIONS}

Figure 1. Location of study lakes in Voyageurs National Park

\section{TABLES}

Table 1. Mercury study lakes in Voyageurs National Park, Minnesota ...................................................................................... 5

Table 2. Surface-water data from selected lakes, Voyageurs National Park, May through September 2000...................................... 6

Table 3. Surface-water data from selected lakes, Voyageurs National Park, May 2001 ............................................................... 7

Table 4. Surface-water data from selected lakes, Voyageurs National Park, July 2001 ................................................................ 8

Table 5. Surface-water data from selected lakes, Voyageurs National Park, September 2001 ....................................................... 9

Table 6. Hypolimnion data from selected lakes, Voyageurs National Park, July 2001 ................................................................... 10

Table 7. Surface-water data from selected lakes, Voyageurs National Park, May 2002 ........................................................... 11

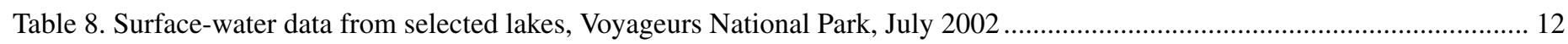

Table 9. Nutrient water chemistry in selected lakes, Voyageurs National Park, May 2002 ........................................................... 13

Table 10. Major-ion water chemistry in selected lakes, Voyageurs National Park, May 2002 ....................................................... 14

Table 11. Quality-assurance results of replicate samples from selected lakes, Voyageurs National Park, 2000-02 ......................... 15

Table 12. Quality-assurance results of blanks from selected lakes, Voyageurs National Park, 2000-02 ........................................... 16

Table 13. Spatial-variation sampling data from selected lakes, Voyageurs National Park, July 2001 and August 2002 ................... 17

\section{CONVERSION FACTORS AND ABBREVIATIONS}

$\begin{array}{lcl}\text { Multiply metric unit } & \text { by } & \text { to obtain inch-pound unit } \\ \text { centimeter }(\mathrm{cm}) & 0.3937 & \text { inch } \\ \text { meter }(\mathrm{m}) & 3.281 & \text { foot } \\ \text { kilometer }(\mathrm{km}) & 0.6214 & \text { mile } \\ \text { square kilometer }\left(\mathrm{km}^{2}\right) & 0.3861 & \text { square mile } \\ \text { Degrees Celsius }\left({ }^{\circ} \mathrm{C}\right) & 1.8 \text { temp }{ }^{\circ} \mathrm{C}+32 & \text { degrees Fahrenheit }\end{array}$

Abbreviated water quality units: chemical concentrations are given in milligrams per liter $(\mathrm{mg} / \mathrm{L})$, micrograms per liter $(\mu \mathrm{g} / \mathrm{L})$, or nanograms per liter (ng/L), units expressing the concentrations of chemical constituents in solution as mass (milligrams, micrograms, or nanograms) per unit volume (liter) of water.

Chemical concentrations in tissues are given in micrograms per gram $(\mu \mathrm{g} / \mathrm{g})$ or nanograms per gram $(\mathrm{ng} / \mathrm{g})$, units expressing the concentrations of chemical constituents as mass within one gram of tissue. 


\title{
Mercury Data from Small Lakes in Voyageurs National Park, Northern Minnesota, 2000-02
}

\author{
by Robert M. Goldstein, Mark E. Brigham, Luke Steuwe, and Michael Menheer
}

\section{ABSTRACT}

Mercury contamination of aquatic ecosystems is a resource concern in Voyageurs National Park. High concentrations of mercury in fish pose a potential risk to organisms that consume large amounts of those fish. During 2000-02, the U.S. Geological Survey measured mercury in water collected from 20 lakes in Voyageurs National Park. Those lakes span a gradient in fish-mercury concentrations, and also span gradients in other environmental variables that are thought to influence mercury cycling. During 2001, near surface methylmercury concentrations ranged from below the method detection limit of 0.04 nanograms per liter (ng/L) to $0.41 \mathrm{ng} / \mathrm{L}$. Near surface total mercury concentrations ranged from $0.34 \mathrm{ng} / \mathrm{L}$ to $3.74 \mathrm{ng} / \mathrm{L}$. Hypolimnetic methylmercury ranged from below detection to $2.69 \mathrm{ng} / \mathrm{L}$, and hypolimnetic total mercury concentrations ranged from $0.34 \mathrm{ng} / \mathrm{L}$ to $7.16 \mathrm{ng} / \mathrm{L}$. During 2002 , near surface methylmercury concentrations ranged from below the method detection limit to $0.46 \mathrm{ng} / \mathrm{L}$, and near surface total mercury ranged from $0.34 \mathrm{ng} / \mathrm{L}$ to $4.81 \mathrm{ng} / \mathrm{L}$. \{ The abstract, table 4, and table 6 were corrected on 14 July 2006. \}

\section{INTRODUCTION}

Mercury is a ubiquitous environmental contaminant and comes from many natural and anthropogenic sources. Atmospheric deposition and naturally occurring minerals are possible sources of mercury, but various land-use and land-cover types also may contribute disproportionate amounts of methylmercury to aquatic ecosystems. Typically, mercury occurs in very low concentrations in surface waters, in both inorganic and organic forms. Inorganic mercury is not particularly toxic, but under certain conditions can be converted to organic mercury (methylmercury), a potent neurotoxin, by bacterially mediated methylation and assimilated by aquatic organisms and magnified in the food chain (Krabbenhoft and Rickert, 1995). Small amounts of inorganic mercury can cause problems in aquatic ecosystems when conditions are suitable for methylation, which primarily occurs as a by-product of bacterially mediated sulfate reduction.

Methlymercury is a potent form of organic mercury and is among the most toxic and widespread contaminants affecting the Nation's aquatic ecosystems (Brumbaugh and others, 2001). Methylation and bioaccumulation of mercury pose toxicological risks to both fish (Wiener and Spry, 1996) and humans (U.S. Environmental Protection Agency, 1997). Because of concerns about methylmercury toxicity, the U.S. Environmental Protection Agency recently reduced the recommended standard for methylmercury in fish tissue from 0.5 milligram per kilogram to 0.3 milligram per kilogram (U.S. Environmental Protection Agency, 2001). Methylmercury contamination also has caused many states, including
Minnesota, to issue human-health advisories for fish consumption.

Mercury contamination of aquatic ecosystems is a resource concern in Voyageurs National Park. High levels of mercury in fish pose a potential risk to organisms that consume large amounts of those fish. This includes both humans and piscivorous (fish-eating) wildlife such as loons, osprey, and eagles.

Concentrations of mercury in northern pike (Esox lucius), a common gamefish species in the Park, vary widely among the lakes in Voyageurs National Park. The estimated concentration of mercury in edible fillets of northern pike (standardized to a length of 55-cm to facilitate comparison among lakes) ranges more than ten-fold, from $0.16 \mu \mathrm{g} / \mathrm{g}$ (wet weight) in Mukooda Lake to 2.3 $\mu \mathrm{g} / \mathrm{g}$ in Ryan Lake (Jeff Jeremiason, Minnesota Pollution Control Agency, written commun., 2002; including unpublished data from 
Gary Glass, University of Minnesota-Duluth).

Tooth and Ryan Lakes in Voyageurs National Park have the greatest concentrations of mercury in fish among Minnesota lakes (using a 55$\mathrm{cm}$ standardized northern pike for comparison). The State of Minnesota recommends no consumption of greater-than-76 cm (30-inch) northern pike from Tooth Lake (Minnesota Department of Health, 1998), which is the single most restrictive advisory the State has issued. Less restrictive fish-consumption advisories exist for many of the Park lakes.

Mercury may be diminishing reproductive success of common loons (Gavia immer) and other piscivorous wildlife in the Park. Recent toxicological investigations suggest that methylmercury contamination of aquatic food webs adversely affects piscivorous fish and wildlife. Methylmercury is the most bioaccumulative and toxic form of mercury that occurs in nature, and is of primary concern when studying mercury cycling. Methylmercury damages the central nervous system, and embryos are the most sensitive life stage of vertebrate organisms (Clarkson, 1992; Scheuhammer, 1991). For example, the effects of methylmercury on birds are much more severe in embryos and chicks than in adults, and low-level dietary exposures that cause no measurable effect in adult birds can significantly impair egg fertility, hatchling survival, and overall reproductive success (Scheuhammer, 1991). Common loon chicks feeding on fish from low $\mathrm{pH}$ lakes in northern Wisconsin lakes have elevated concentrations of mercury in their blood (Meyer and others, 1995), and diminished reproduction has been observed in some of these lakes (Meyer and others, 1998). In Ontario, Scheuhammer and Blancher
(1994) estimate that 30 percent of the lakes had prey-size fish with mercury levels high enough to impair reproduction of common loons.

Based on relations between predator and prey fish species, it is likely that some Park lakes with high levels of mercury in gamefish have mercury levels of $0.3-0.4 \mu \mathrm{g} / \mathrm{g}$ in prey species, such as young yellow perch (James Wiener, U.S. Geological Survey, La Crosse, Wisc., oral commun., 2000). These levels in prey fish species have been shown to adversely effect loon reproduction (Barr, 1986), and may adversely affect other piscivores such as gamefish. Friedmann and others (1996) found that testicular development and immune function were inhibited by mercury levels of $100 \mathrm{ng} / \mathrm{g}(0.1 \mu \mathrm{g} / \mathrm{g})$ in food, a dietary methylmercury concentration available to piscivorous fish in many North American waters.

The processes and factors causing large variability in fish-mercury levels among Voyageurs National Park lakes have not yet been identified. It is likely that within this small geographic area, atmospheric mercury deposition is uniform. Greater than 90 percent of the mercury that enters remote lakes is derived from the atmospheric deposition (Swain and others, 1992). Large inter-lake variability in fish-mercury levels indicates that factors other than mercury deposition control mercury accumulation in fish.

Many factors affect mercury cycling and bioaccumulation, although the cycling is complex and not completely understood. Variability in lake trophic status, food web structure, size, and morphometry all influence, or at least correlate with, mercury levels in fish (Wiener and Spry, 1996). Low-alkalinity, low-pH lakes, such as those found in the
Park, often have fish with high fishmercury levels (Swain and Helwig, 1989; Wiener and Spry, 1996). Lakes that have large watershed-area to lake-surface-area ratios tend to have greater mercury accumulation rates, because some fraction of mercury deposited in upland areas is delivered to the lake (Swain, and others, 1992). Wetlands can be net sinks of total mercury, but net sources of methylmercury (St. Louis and others, 1994).

Analysis of mercury in lake-sediment cores from mid North American lakes has shown that mercury accumulation rates have almost tripled since pre-industrial times (Swain and others, 1992), and this increase has been attributed to atmospheric transport and deposition from industrial sources. Recent measurements of methylmercury in lakesediment cores indicates that the percentage of total mercury that is in the methylmercury form has increased substantially since the mid 1900's in some lakes-including four lakes in Voyageurs National Park (Engstrom and others, 1999).

The U.S. Geological Survey, in cooperation with the National Park Service, and the Minnesota Pollution Control Agency conducted a study to measure seasonality and spatial variability of total mercury and methylmercury concentrations in 20 lakes in Voyageurs National Park. This report presents the data collected from this study during 2000-02.

\section{METHODS}

Mercury occurs in low concentration in water, and may be present in low concentrations in the atmosphere and on surfaces near or in the sampling and processing environment. Precautions are necessary during sample collection such that exposure of the water sample to different collection containers, humans, and the 
atmosphere must be minimized. For this study, two-person ultra-clean sampling procedures (U.S. Environmental Protection Agency (USEPA) Method 1669 (U.S. Environmental Protection Agency, 1996)) were used to collect the water samples analyzed for mercury constituents. The collectors wore disposable shoulder-length polyethylene gloves and wrist-length latex gloves. Sample bottles were contained with double polyethylene bags, and any materials and containers that contacted the sample water were cleaned following procedures outlined by Olson and DeWild (1999).

Twenty lakes (fig. 1) were sampled in May, July, and September during 2001 and during May and July 2002 for determination of total mercury and methylmercury from epilimnetic (near surface) waters. The same lakes were sampled for major ions, nutrients, and organic carbon once. Water also was collected from the hypolimnion (near bottom) waters once during midsummer (July 2001). This sampling was conducted using trace-metalclean protocols that have been demonstrated to not contaminate samples. Total mercury, methylmercury, elemental mercury, and total organic carbon were analyzed at the USGSWRD mercury laboratory in Middleton, Wisconsin (the "Laboratory" hereinafter). This laboratory is a class-100 clean-room facility, and quality-assurance procedures are available at URL

http://infotrek.er.usgs.gov/doc/mercury/. Total mercury concentrations were determined using USEPA method 1631 (U.S. Environmental Protection Agency, 1999) as modified by Olson and DeWild (1999). Methylmercury concentrations were determined using methods described in De Wild and others (2002). Total organic carbon concentrations were determined using a carbon analyzer (model 1010, OI Analytical, College Station Texas) using Standard Method 5301D (American Public Health Association and others, 1998). Major ions were analyzed at the USGS National Water Quality Laboratory in Lakewood, Colorado. A vertical profile of field parameters (specific conductance, temperature, dissolved oxygen, $\mathrm{pH}$, and Secchi transparency) was obtained at each sampling. Twenty percent of all field-submitted samples were quality-control samples (field blanks and replicates) and in-lake spatial variability samples.

The 20 study lakes (fig. 1; table 1) were selected to maximize withinPark gradients in water chemistry, fish-mercury levels, and landscape features. Use of data was maximized from past and ongoing studies in the Park. Each of the study lakes has been sampled for routine water-quality parameters. Nearly all have been sampled for gamefish that were analyzed for mercury (typically northern pike; some walleye and muskellunge; cited above) and age- 1 yellow perch, and this work is planned to continue under State programs and MPCA-USGS cooperative studies.

Four lakes were sampled for total mercury, and methylmercury as a pilot study during May-September 2000 (Little Trout, Locator, Shoepack, and Tooth Lakes) (table 2). These lake bottoms were cored, and sediment-mercury accumulation rates were determined, and three of these four lakes had methylmercury analyzed in sediment profiles (Engstrom and others, 1999).

Surface-water sampling during 2001 was conducted during May, July, and September. Concentrations of methylmercury, total mercury, and other water-chemistry parameters in surface water for May, July, and Sep- tember 2001 are presented in tables 3,4 , and 5 , respectively. The results of the July hypolimnetic samples are presented in table 6 .

For the 2002 sampling season, two sets of samples were collected. Methylmercury and total mercury concentrations from surface-water samples collected in May and July of 2002 are presented in tables 7 and 8 . In addition, during the May sampling, samples were collected for nutrients (table 9) and for major ions (table 10).

Two types of quality assurance samples were collected: replicate samples and blank samples. Replicate samples were additional samples collected at the same location and with the same methods as the regular environmental sample. Blank samples were collected by processing low-mercury de-ionized water (obtained from the USGS Wisconsin District Mercury Laboratory) as if it were an environmental sample. This process involved pouring blank water into sample containers at the field site (field blank, table 12) or at the office (office blank, table 12). During July 2001, when hypolimnetic waters were sampled with a peristaltic pump, pump blanks (table 12) were obtained after rinsing the pump tubing with 1 percent hydrochloric acid (standard procedure after sampling each site). Quality assurance sample results are presented in tables 11 and 12.

To assess spatial variability of methylmercury and total mercury concentrations, sets of spatial-variability samples were collected from Locator Lake during July 2001 and Net Lake during August 2002. Surface samples were collected from 5-6 points across each lake (table 13). The data also can be found on the USGS web site (U.S. Geological Survey, 2003). 

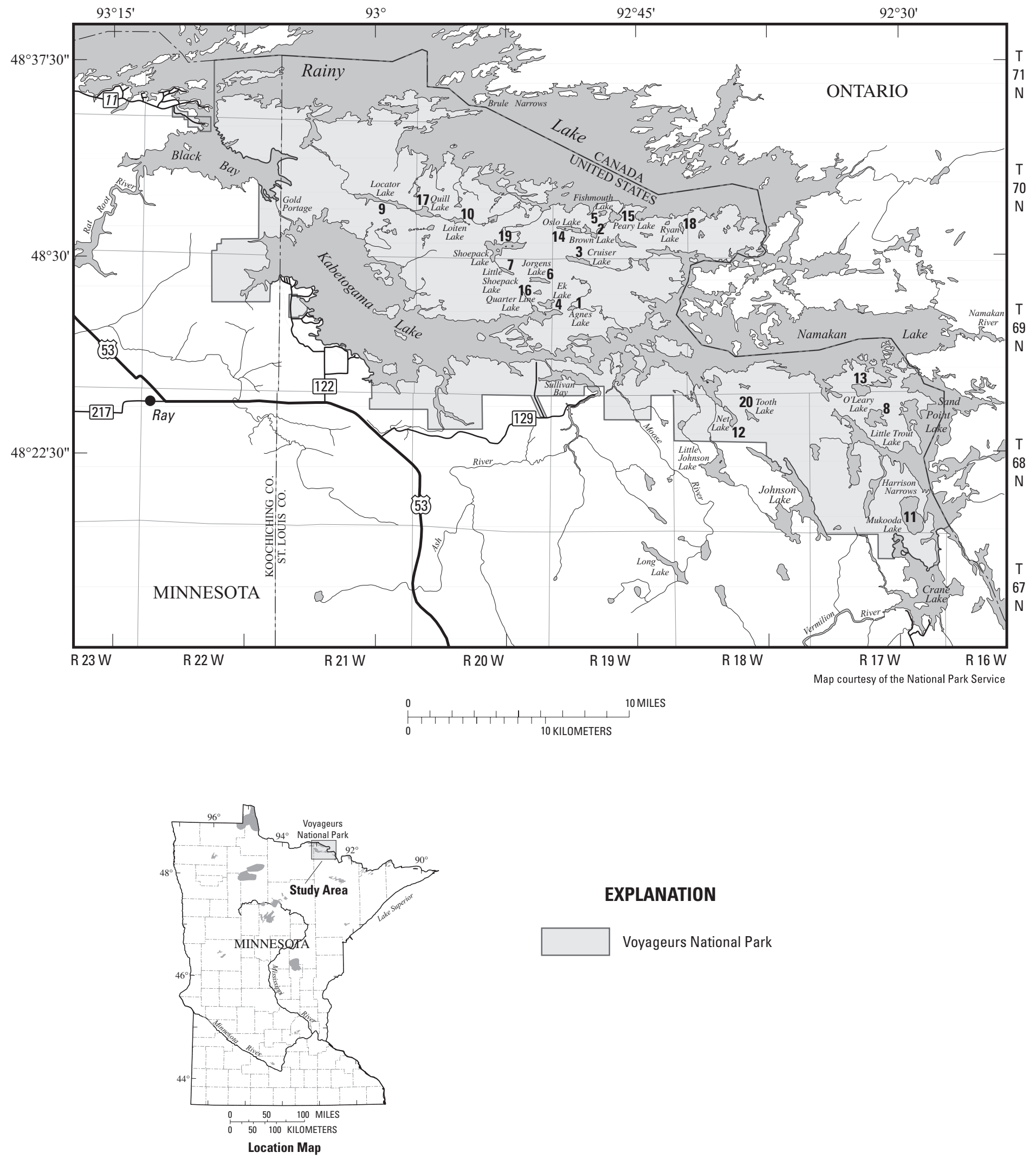

EXPLANATION

Voyageurs National Park

Figure 1. Location of study lakes in Voyageurs National Park (Number corresponds to lake number in table 1). 
Table 1. Mercury study lakes in Voyageurs National Park, Minnesota

[USGS, U.S. Geological Survey; Modified from Kallemeyn and others, 2003]

\begin{tabular}{cllcr}
\hline $\begin{array}{c}\text { Lake number } \\
\text { (figure 1) }\end{array}$ & USGS Station number & Lake & $\begin{array}{c}\text { Surface area } \\
\text { (hectares) }\end{array}$ & $\begin{array}{c}\text { Mean depth } \\
\text { (meters) }\end{array}$ \\
\hline 1 & 482805092484101 & Agnes & 12.9 & 2.5 \\
2 & 483059092474501 & Brown & 30.8 & 4.2 \\
3 & 482958092484501 & Cruiser & 46.5 & 13.2 \\
4 & 482812092500801 & Ek & 36.0 & 2.4 \\
5 & 483142092465201 & Fishmouth & 12.9 & 3.8 \\
6 & 482903092504701 & Jorgens & 24.7 & 3.0 \\
7 & 482924092525101 & Little Shoepack & 22.7 & 2.5 \\
8 & 482353092311901 & Little Trout & 96.7 & 13.0 \\
9 & 483226092001401 & Locator & 56.7 & 8.1 \\
10 & 483139092553801 & Loiten & 36.6 & 7.8 \\
11 & 482018092292001 & Mukooda & 305 & 12.2 \\
12 & 482358092392701 & Net & 43.7 & 2.0 \\
13 & 482459092320101 & O'Leary & 78.5 & 7.1 \\
14 & 483103092482501 & Oslo & 42.5 & 4.7 \\
15 & 483129092462001 & Peary & 45.3 & 2.6 \\
16 & 482838092504001 & Quarter Line & 8.3 & 3.1 \\
17 & 483157092565701 & Quill & 34.4 & 6.9 \\
18 & 483109092422601 & Ryan & 14.2 & 2.1 \\
19 & 482951092531601 & Shoepack & 123.8 & 2.9 \\
20 & 482355092383601 & Tooth & 23.5 & 5.9 \\
\hline
\end{tabular}




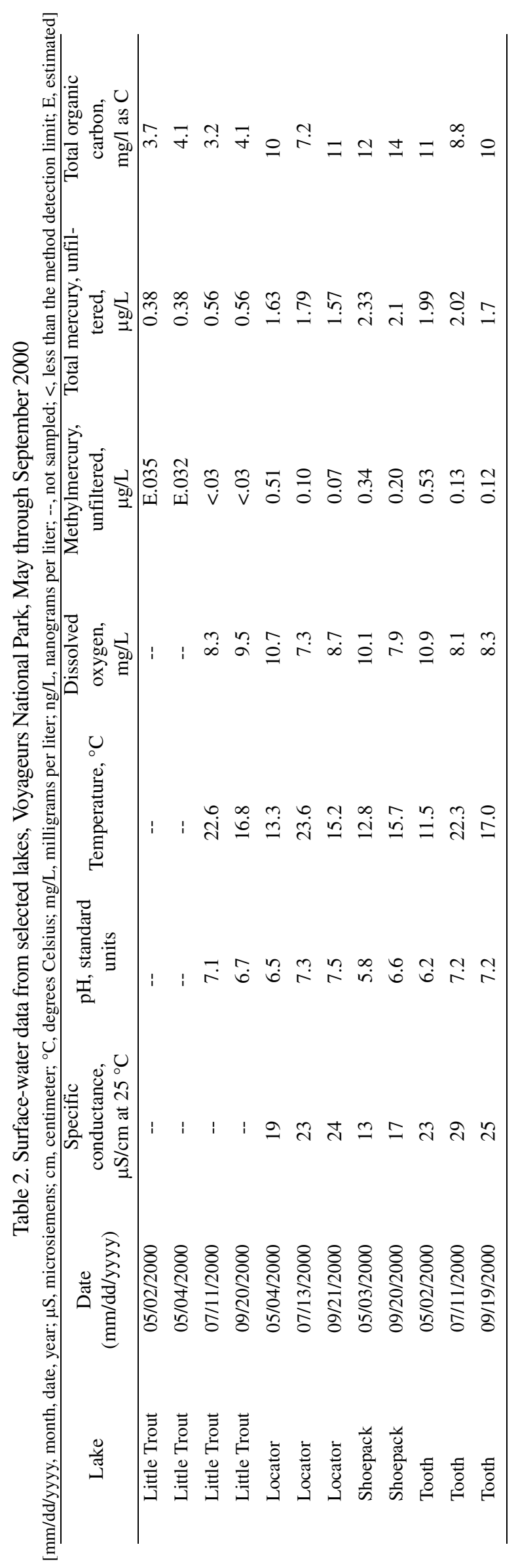


Table 3. Surface-water data from selected lakes, Voyageurs National Park, May 2001

$\underline{\left[\mu \mathrm{S}, \text { microsiemens; } \mathrm{cm} \text {, centimeters; }{ }^{\circ} \mathrm{C} \text {, degrees Celsius; } \mathrm{mg} / \mathrm{L} \text {, milligrams per liter; ng/L, nanograms per liter; --, not sampled; <, less than the method detection limit }\right]}$

\begin{tabular}{|c|c|c|c|c|c|c|c|}
\hline Lake & $\begin{array}{c}\text { Specific } \\
\text { conductance, } \\
\mu \mathrm{S} / \mathrm{cm} \text { at } 25^{\circ} \mathrm{C}\end{array}$ & $\begin{array}{c}\mathrm{pH}, \text { standard } \\
\text { units }\end{array}$ & $\begin{array}{c}\text { Temperature, } \\
{ }^{\circ} \mathrm{C}\end{array}$ & $\begin{array}{l}\text { Dissolved } \\
\text { oxygen, } \\
\text { mg/L }\end{array}$ & $\begin{array}{c}\text { Methylmercury, } \\
\text { unfiltered, } \\
\text { ng/L }\end{array}$ & $\begin{array}{c}\text { Total mercury, } \\
\text { unfiltered, } \\
\text { ng/L }\end{array}$ & $\begin{array}{l}\text { Total organic } \\
\text { carbon, } \\
\mathrm{mg} / \mathrm{L} \text { as } \mathrm{C}\end{array}$ \\
\hline Agnes & 7 & 6.2 & 13.9 & 6.5 & 0.26 & 2.99 & 16 \\
\hline Brown & 16 & 7.0 & 16.5 & 9.5 & 0.09 & 2.05 & 8.8 \\
\hline Cruiser & 13 & -- & 14.5 & 11.0 & $<0.04$ & 0.44 & 3.1 \\
\hline Ek & 18 & 6.8 & 14.5 & 8.5 & 0.08 & 1.97 & 12 \\
\hline Fishmouth & 15 & -- & 17.1 & 9.2 & 0.12 & 1.49 & 7.3 \\
\hline Jorgens & 14 & 6.8 & 14.3 & 8.2 & 0.12 & 2.07 & 9.4 \\
\hline Little Shoepack & 14 & 6.8 & 14.4 & 8.9 & 0.15 & 2.54 & 11 \\
\hline Little Trout & 29 & 7.5 & 13.9 & 10.0 & 0.05 & 0.86 & 4.0 \\
\hline Locator & 14 & 6.7 & 14.6 & 10.8 & 0.15 & 2.72 & 11 \\
\hline Loiten & 14 & 6.9 & 14.7 & 10.9 & 0.13 & 2.22 & 9.0 \\
\hline Mukooda & 48 & 7.1 & 12.2 & 11.0 & $<0.04$ & 0.55 & 5.2 \\
\hline Net & 23 & 6.5 & 13.7 & 8.7 & 0.40 & 3.52 & 17 \\
\hline O'Leary & 61 & 7.5 & 14.0 & 10.0 & $<0.04$ & 0.78 & 6.9 \\
\hline Oslo & 15 & 7.2 & 16.6 & 8.7 & 0.16 & 2.16 & 10 \\
\hline Peary & 17 & 7.3 & 16.7 & 9.0 & 0.13 & 1.77 & 9.4 \\
\hline Quarter Line & 13 & 6.4 & 13.8 & 7.6 & 0.12 & 3.33 & 18 \\
\hline Quill & 14 & 6.8 & 15.3 & 10.8 & 0.08 & 2.38 & 9.2 \\
\hline Ryan & 18 & -- & 16.5 & 8.3 & 0.24 & 3.52 & 12 \\
\hline Shoepack & 13 & 6.6 & 14.1 & 8.0 & 0.24 & 2.85 & 14 \\
\hline Tooth & 20 & 6.6 & 14.3 & 11.1 & 0.17 & 3.74 & 11 \\
\hline
\end{tabular}


Table 4. Surface-water data from selected lakes, Voyageurs National Park, July 2001

\begin{tabular}{|c|c|c|c|c|c|c|c|c|}
\hline Lake & $\begin{array}{c}\text { Specific } \\
\text { conductance, } \\
\mu \mathrm{S} / \mathrm{cm} \text { at } 25^{\circ} \mathrm{C}\end{array}$ & $\begin{array}{c}\mathrm{pH}, \\
\text { standard } \\
\text { units }\end{array}$ & $\begin{array}{c}\text { Temperature, } \\
{ }^{\circ} \mathrm{C}\end{array}$ & $\begin{array}{c}\text { Secchi disk } \\
\text { transparency, } \\
\text { meters }\end{array}$ & $\begin{array}{c}\text { Dissolved } \\
\text { oxygen, mg/L }\end{array}$ & $\begin{array}{l}\text { Methylmercury, } \\
\text { unfiltered, } \\
\text { ng/L }\end{array}$ & $\begin{array}{c}\text { Total mercury, } \\
\text { unfiltered, } \\
\text { ng/L }\end{array}$ & $\begin{array}{c}\text { Total organic } \\
\text { carbon, } \\
\mathrm{mg} / \mathrm{L} \text { as } \mathrm{C}\end{array}$ \\
\hline Agnes & 18 & 6.3 & 24.5 & 0.9 & 5.2 & 0.41 & 2.74 & 17 \\
\hline Brown & 13 & 7.1 & 25.0 & 2.0 & 9.0 & 0.12 & 1.83 & 9.3 \\
\hline Cruiser & 12 & 6.6 & 23.3 & 5.5 & 8.1 & $<0.04$ & 0.39 & 3.6 \\
\hline Ek & 29 & 6.9 & 25.2 & 1.6 & 8.3 & 0.28 & 1.92 & 13 \\
\hline Fishmouth & 21 & 7.6 & 24.5 & 3.9 & 8.2 & 0.04 & 1.08 & 7.9 \\
\hline Jorgens & 24 & 6.5 & 24.6 & 1.9 & 8.4 & 0.08 & 1.74 & 9.6 \\
\hline Little Shoepack & 15 & 6.7 & 25.4 & 2.1 & 7.7 & 0.15 & 1.99 & 11 \\
\hline Little Trout & 27 & 7.7 & 24.6 & 4.5 & 7.9 & $<0.04$ & 0.43 & 4.2 \\
\hline Locator & 16 & 6.4 & 26.7 & 2.5 & 10.5 & 0.08 & 2.12 & 11 \\
\hline Loiten & 24 & 6.9 & 26.4 & 3.9 & 8.2 & 0.10 & 2.02 & 8.6 \\
\hline Mukooda & 44 & 7.3 & 23.9 & 4.2 & 8.2 & $<0.04$ & 0.52 & 5.4 \\
\hline $\mathrm{Net}^{1}$ & -- & -- & -- & -- & -- & -- & -- & -- \\
\hline O'Leary & 55 & 8.0 & 26.6 & 4.9 & 8.3 & 0.05 & 0.68 & 7.0 \\
\hline Oslo & 13 & 7.5 & 24.8 & 2.0 & 9.1 & 0.11 & 2.74 & 12 \\
\hline Peary & 25 & 6.9 & 24.7 & 2.3 & 7.9 & 0.2 & 1.60 & 10 \\
\hline Quarter Line & 23 & 6.8 & 26.2 & 1.4 & 8.2 & 0.17 & 2.79 & 18 \\
\hline Quill & 23 & 7.2 & 27.0 & 3.0 & 7.7 & 0.09 & 1.81 & 9.0 \\
\hline Ryan & 25 & 7.0 & 24.9 & 2.8 & 8.1 & 0.15 & 2.82 & 13 \\
\hline Shoepack & 12 & 6.2 & 24.5 & 1.6 & 8.0 & 0.27 & 2.56 & 14 \\
\hline Tooth & 29 & 7.1 & 26.3 & 2.5 & 7.9 & 0.16 & 2.48 & 11 \\
\hline
\end{tabular}

${ }^{1}$ Net Lake had drained due to the failure of a beaver dam.

Note: This table shows corrected values for Shoepack Lake, methylmercury and total mercury concentrations. An Erratum to this report (14 July 2006) explains the error in the original report. 
Table 5. Surface-water data from selected lakes, Voyageurs National Park, September 2001

$\left[\mu \mathrm{S}\right.$, microsiemens; $\mathrm{cm}$, centimeter; ${ }^{\circ} \mathrm{C}$, degrees Celsius; $\mathrm{mg} / \mathrm{L}$, milligrams per liter; ng/L, nanograms per liter; --, not sampled; <, less than the method detection limit]

\begin{tabular}{|c|c|c|c|c|c|c|c|c|}
\hline Lake & $\begin{array}{c}\text { Specific } \\
\text { conductance, } \\
\mu \mathrm{S} / \mathrm{cm} \text { at } 25^{\circ} \mathrm{C}\end{array}$ & $\begin{array}{c}\mathrm{pH}, \\
\text { standard } \\
\text { units }\end{array}$ & $\begin{array}{c}\text { Temperature, } \\
{ }^{\circ} \mathrm{C}\end{array}$ & $\begin{array}{c}\text { Secchi disk } \\
\text { transparency, } \\
\text { meters }\end{array}$ & $\begin{array}{l}\text { Dissolved } \\
\text { oxygen, } \\
\text { mg/L }\end{array}$ & $\begin{array}{l}\text { Methylmercury, } \\
\text { unfiltered, } \\
\text { ng/L }\end{array}$ & $\begin{array}{l}\text { Total mercury, } \\
\text { unfiltered, } \\
\text { ng/L }\end{array}$ & $\begin{array}{c}\text { Total } \\
\text { organic carbon, } \\
\mathrm{mg} / \mathrm{L} \text { as } \mathrm{C}\end{array}$ \\
\hline Agnes & 21 & 5.7 & 15.3 & 0.9 & 0.23 & 0.30 & 2.43 & 19 \\
\hline Brown & 20 & 6.7 & 17.3 & 2.2 & 8.3 & $<0.04$ & 1.84 & 10 \\
\hline Cruiser & 18 & 7.5 & 18.4 & 6.5 & 9.0 & $<0.04$ & 0.46 & 3.8 \\
\hline $\mathrm{Ek}$ & 28 & 7.2 & 18.3 & 1.8 & 8.3 & 0.09 & 1.66 & 13 \\
\hline Fishmouth & 22 & 7.0 & 17.5 & 2.7 & 8.3 & 0.05 & 0.97 & 8.4 \\
\hline Jorgens & 24 & 6.6 & 17.7 & 2.5 & 7.5 & 0.21 & 1.74 & 11 \\
\hline Little Shoepack & 20 & 7.1 & 18.1 & 1.4 & 8.4 & 0.27 & 2.01 & 13 \\
\hline Little Trout & 38 & 7.7 & 18.4 & 6.2 & 8.7 & $<0.04$ & 0.41 & 4.5 \\
\hline Locator & 24 & 6.8 & 18.6 & 2.4 & 7.8 & 0.12 & 2.24 & 12 \\
\hline Loiten & 24 & 6.8 & 18 & 3.6 & 8.0 & 0.08 & 2.15 & 11 \\
\hline Mukooda & 57 & 8.0 & 18.2 & 3.5 & 8.7 & $<0.04$ & 0.34 & 5.9 \\
\hline $\mathrm{Net}^{1}$ & -- & -- & -- & -- & -- & -- & -- & -- \\
\hline O'Leary & 68 & 7.5 & 18.1 & 4.7 & 8.3 & $<0.04$ & 0.47 & 7.3 \\
\hline Oslo & 18 & 6.7 & 17.3 & 1.8 & 8.0 & 0.22 & 2.14 & 12 \\
\hline Peary & 25 & 7.2 & 17.3 & 1.7 & 7.6 & 0.24 & 1.43 & 11 \\
\hline Quarter Line & 22 & 6.7 & 17.1 & 1.3 & 7.4 & 0.30 & 2.68 & 20 \\
\hline Quill & 26 & 7.2 & 18.6 & 3.3 & 8.2 & 0.08 & 1.75 & 10 \\
\hline Ryan & 25 & 6.6 & 17.8 & 2.0 & 8.0 & 0.32 & 2.85 & 13 \\
\hline Shoepack & 19 & 6.5 & 17.6 & 0.9 & 8.1 & 0.39 & 2.80 & 18 \\
\hline Tooth & 30 & 7.0 & 17.4 & 2.1 & 8.4 & 0.18 & 2.33 & 12 \\
\hline
\end{tabular}

${ }^{1}$ Net Lake had drained due to the failure of a beaver dam. 
Table 6. Hypolimnion (bottom water) data from selected lakes, Voyageurs National Park, July 2001

\begin{tabular}{|c|c|c|c|c|c|c|c|}
\hline Lake & $\begin{array}{l}\text { Specific conduc- } \\
\text { tance, } \\
\mu \mathrm{S} / \mathrm{cm} \text { at } 25^{\circ} \mathrm{C}\end{array}$ & $\begin{array}{c}\mathrm{pH}, \text { standard } \\
\text { units }\end{array}$ & $\begin{array}{c}\text { Temperature, } \\
{ }^{\circ} \mathrm{C}\end{array}$ & $\begin{array}{l}\text { Dissolved oxy- } \\
\text { gen, } \\
\mathrm{mg} / \mathrm{L}\end{array}$ & $\begin{array}{l}\text { Methylmercury, } \\
\text { unfiltered, } \\
\text { ng/L }\end{array}$ & $\begin{array}{l}\text { Total mercury, } \\
\text { unfiltered, } \\
\text { ng/L }\end{array}$ & $\begin{array}{c}\text { Total organic } \\
\text { carbon, } \\
\text { mg/L as C }\end{array}$ \\
\hline Agnes & 32 & 5.9 & 10.7 & 0.2 & 1.81 & 5.98 & 17 \\
\hline Brown & 14 & 6.3 & 10.5 & 0.8 & 0.17 & 1.86 & 8.3 \\
\hline Cruiser & 11 & 5.8 & 5.1 & 5.2 & $<0.04$ & 0.34 & 3.2 \\
\hline Ek & 68 & 6.3 & 11.3 & 0.2 & 0.75 & 4.94 & 13 \\
\hline Fishmouth & 25 & 7.2 & 13.3 & 6.7 & 0.05 & 0.95 & 8.0 \\
\hline Jorgens & 31 & 6.0 & 16.1 & 0.3 & 0.14 & 2.15 & 9.6 \\
\hline Little Shoepack & 21 & 6.0 & 16.3 & 0.6 & 2.69 & 7.16 & 12 \\
\hline Little Trout & 24 & 7.0 & 5.1 & 8.3 & $<0.04$ & 0.44 & 3.5 \\
\hline Locator & 16 & 5.8 & 14.2 & 6.8 & 0.11 & 2.08 & 9.8 \\
\hline Loiten & 26 & 5.9 & 6.2 & 2.3 & 0.28 & 1.60 & 7.7 \\
\hline Mukooda & 40 & 6.9 & 6.3 & 4.7 & $<0.04$ & 0.64 & 4.7 \\
\hline $\mathrm{Net}^{1}$ & -- & -- & -- & -- & -- & -- & -- \\
\hline O'Leary & 55 & 7.0 & 7.5 & 0.9 & 0.10 & 0.66 & 6.8 \\
\hline Oslo & 13 & 6.4 & 14.9 & 2.6 & 0.14 & 1.86 & 9.8 \\
\hline Peary & 44 & 6.4 & 15.0 & 0.4 & 0.15 & 1.52 & 10 \\
\hline Quarter Line & 40 & 5.8 & 8.3 & 0.2 & 0.18 & 3.11 & 18 \\
\hline Quill & 27 & 6.1 & 5.2 & 0.5 & 0.17 & 1.40 & 8.0 \\
\hline Ryan & 25 & 6.5 & 18.0 & 4.0 & 0.17 & 2.73 & 13 \\
\hline Shoepack & 13 & 5.7 & 19.6 & 3.7 & 1.02 & 3.82 & 14 \\
\hline Tooth & 30 & 5.9 & 5.1 & 0.3 & 0.17 & 2.57 & 9.5 \\
\hline
\end{tabular}

${ }^{1}$ Net Lake had drained due to the failure of a beaver dam.

Note: This table shows corrected values for Shoepack Lake, methylmercury and total mercury concentrations. An Erratum to this report (14 July 2006) explains the error in the original report. 
Table 7. Surface-water data from selected lakes, Voyageurs National Park, May 2002

$\left[\mu \mathrm{S}\right.$, microsiemens; $\mathrm{cm}$, centimeter; ${ }^{\circ} \mathrm{C}$, degrees Celsius; $\mathrm{mg} / \mathrm{L}$, milligrams per liter; $\mathrm{ng} / \mathrm{L}$, nanograms per liter, --not sampled; <, less than the method detection limit]

\begin{tabular}{|c|c|c|c|c|c|c|c|c|c|}
\hline Lake & $\begin{array}{c}\text { Specific } \\
\text { conductance, } \\
\mu \mathrm{S} / \mathrm{cm} \text { at } 25^{\circ} \mathrm{C}\end{array}$ & $\begin{array}{c}\text { Field pH, } \\
\text { standard } \\
\text { units }\end{array}$ & $\begin{array}{c}\text { Laboratory } \\
\mathrm{pH}, \text { standard } \\
\text { units }\end{array}$ & $\begin{array}{c}\text { Temperature, } \\
\text { degrees C }\end{array}$ & $\begin{array}{l}\text { Secchi disk } \\
\text { transparency, } \\
\text { meters }\end{array}$ & $\begin{array}{c}\text { Dissolved } \\
\text { oxygen, } \\
\text { mg/L }\end{array}$ & $\begin{array}{l}\text { Bicarbonate, } \\
\text { field, } \\
\mathrm{mg} / \mathrm{L} \text { as } \mathrm{HCO}_{3}\end{array}$ & $\begin{array}{c}\text { Methyl- } \\
\text { mercury, } \\
\text { unfiltered, } \\
\text { ng/L }\end{array}$ & $\begin{array}{c}\text { Total } \\
\text { mercury, } \\
\text { unfiltered, } \\
\text { ng/L }\end{array}$ \\
\hline Agnes & 19 & -- & 6.5 & 7.9 & 1.0 & 9.3 & 5 & 0.33 & 3.07 \\
\hline Brown & 13 & 6.4 & 7.1 & 6.7 & 2.6 & 11 & 7 & 0.15 & 1.83 \\
\hline Cruiser $^{1}$ & -- & -- & -- & -- & -- & -- & -- & -- & -- \\
\hline Ek & 26 & 6.9 & 7.1 & 8.6 & 2.0 & 10.8 & 14 & 0.08 & 1.74 \\
\hline Fishmouth & 17 & 6.6 & 6.9 & 7.9 & 3.0 & 11.3 & 14 & 0.07 & 1.00 \\
\hline Jorgens & 20 & 6.7 & 7.0 & 9.5 & 2.2 & 10.6 & 9 & 0.11 & 1.82 \\
\hline Little Shoepack & 20 & -- & 6.6 & 7.9 & 1.5 & 10.6 & 12 & 0.12 & 2.08 \\
\hline Little Trout & 33 & 7.2 & 7.2 & 5.8 & 3.1 & 11.7 & 18 & $<0.04$ & 0.38 \\
\hline Locator & 22 & 6.9 & 6.8 & 5.5 & 2.5 & 10.5 & 8 & 0.20 & 2.26 \\
\hline Loiten & 22 & 6.7 & 6.9 & 5.8 & 3.5 & 10.8 & 8 & 0.20 & 2.07 \\
\hline Mukooda & 50 & 7.5 & 7.5 & 5.1 & 3.5 & 11.9 & 32 & $<0.04$ & 0.34 \\
\hline Net & 29 & 6.8 & 6.9 & 7.8 & 1.4 & 11.2 & 14 & 0.18 & 3.05 \\
\hline O'Leary & 62 & 7.7 & 7.5 & 6.0 & 3.0 & 11.8 & 38 & 0.06 & 0.55 \\
\hline Oslo & 12 & 6.6 & 6.6 & 6.2 & 2.0 & 11.4 & 7 & 0.30 & 2.26 \\
\hline Peary & 18 & 6.5 & 7.0 & 7.7 & 3.2 & 10.0 & 14 & 0.08 & 1.51 \\
\hline Quarter line & 22 & -- & 6.7 & 9.2 & 1.5 & 11.1 & 7 & 0.22 & 3.02 \\
\hline Quill & 23 & 6.7 & 7.2 & 6.1 & 3.0 & 10.5 & 8 & 0.25 & 1.66 \\
\hline Ryan & 20 & 6.6 & 6.9 & 8.1 & 2.6 & 11.1 & 10 & 0.21 & 2.74 \\
\hline Shoepack & 19 & 6.2 & 6.6 & 7.3 & 1.0 & 10.5 & 8 & 0.20 & 3.24 \\
\hline Tooth & 22 & 6.7 & 7.1 & 6.3 & 2.3 & 11.4 & 16 & 0.25 & 2.20 \\
\hline
\end{tabular}

${ }^{1}$ Cruiser Lake was not included in this study because of consistently low mercury and methylmercury concentrations, and because a collaborative fish study did not sample fish from this lake. 
Table 8. Surface-water data from selected lakes, Voyageurs National Park, July 2002

[ $\mu \mathrm{S}$, microsiemens, $\mathrm{cm}$, centimeters; ${ }^{\circ} \mathrm{C}$, degrees Celsius; mg/L, milligrams per liter; ng/L, nanograms per liter; --, not sampled; <, less than the detection limit]

\begin{tabular}{|c|c|c|c|c|c|c|c|}
\hline Lake & $\begin{array}{c}\text { Specific } \\
\text { conductance, } \\
\mu \mathrm{S} / \mathrm{cm} \text { at } 25^{\circ} \mathrm{C}\end{array}$ & $\begin{array}{c}\mathrm{pH}, \text { standard } \\
\text { units }\end{array}$ & $\begin{array}{c}\text { Temperature, } \\
{ }^{\circ} \mathrm{C}\end{array}$ & $\begin{array}{c}\text { Secchi disk } \\
\text { transparency, } \\
\text { meters }\end{array}$ & $\begin{array}{l}\text { Dissolved } \\
\text { oxygen, } \\
\text { mg/L }\end{array}$ & $\begin{array}{l}\text { Methylmercury, } \\
\text { unfiltered, ng/L }\end{array}$ & $\begin{array}{l}\text { Total mercury, } \\
\text { unfiltered, ng/L }\end{array}$ \\
\hline Agnes & 17 & 6.2 & 29.2 & 0.9 & 7.1 & 0.46 & 3.67 \\
\hline Brown & 9 & 6.6 & 26.6 & 1.7 & 7.4 & 0.13 & 3.07 \\
\hline Cruiser $^{1}$ & -- & -- & -- & -- & -- & -- & -- \\
\hline Ek & 24 & 6.6 & 28.3 & 1.5 & 7.9 & 0.20 & 2.66 \\
\hline Fishmouth & -- & -- & -- & 2.6 & -- & 0.07 & 1.73 \\
\hline Jorgens & 21 & 6.1 & 27.4 & 1.9 & 7.7 & 0.13 & 2.86 \\
\hline Little Shoepack & 12 & 6.1 & 27.2 & 1.5 & 7.1 & 0.18 & 3.22 \\
\hline Little Trout & 32 & 7.5 & 26.8 & 6.0 & 7.3 & $<0.04$ & 0.51 \\
\hline Locator & -- & -- & -- & 1.7 & -- & 0.16 & 3.12 \\
\hline Loiten & 14 & 6.6 & 28.1 & 2.4 & 7.7 & 0.4 & 3.06 \\
\hline Mukooda & 56 & 7.4 & 26.4 & 4.3 & 8.1 & $<0.04$ & 0.43 \\
\hline Net & 37 & 6.3 & 26.9 & 1.0 & 7.4 & 0.41 & 3.67 \\
\hline O’Leary & 70 & 8.1 & 27.2 & 4.5 & 7.5 & 0.05 & 0.72 \\
\hline Oslo & 7 & 6.9 & 27.7 & 1.7 & 7.4 & 0.17 & 3.53 \\
\hline Lake & 22 & 6.0 & 26.6 & 1.8 & 8.3 & 0.21 & 2.38 \\
\hline Quarter Line & 20 & 6.1 & 28.0 & 1.2 & 7.1 & 0.20 & 4.32 \\
\hline Quill & 14 & 7.1 & 28.2 & 2.1 & 7.5 & 0.13 & 2.69 \\
\hline Ryan & 14 & 6.5 & 28.1 & 1.5 & 6.8 & 0.22 & 4.81 \\
\hline Shoepack & 20 & 6.0 & 26.1 & 1.0 & 7.2 & 0.34 & 3.83 \\
\hline Tooth & 30 & 6.7 & 27.0 & 2.5 & 7.2 & 0.23 & 3.26 \\
\hline
\end{tabular}

${ }^{1}$ Cruiser Lake was not included in this study because of consistently low mercury and methylmercury concentrations, and because a collaborative fish study did not sample fish from this lake. 
Table 9. Nutrient water chemistry in selected lakes, Voyageurs National Park, May 2002

[mg/L, milligrams per liter; --, not sampled; <, less than the method detection limit; E, estimated]

\begin{tabular}{|c|c|c|c|c|c|c|c|c|}
\hline Lake & $\begin{array}{c}\text { Dissolved nitrite } \\
\text { nitrogen, } \\
\mathrm{mg} / \mathrm{L} \text { as } \mathrm{N}\end{array}$ & $\begin{array}{l}\text { Dissolved nitrite } \\
\text { plus nitrate } \\
\text { nitrogen, } \\
\text { mg/Las } \mathrm{N}\end{array}$ & $\begin{array}{c}\text { Dissolved } \\
\text { ammonia } \\
\text { nitrogen, } \\
\mathrm{mg} / \mathrm{L} \text { as N, }\end{array}$ & $\begin{array}{c}\text { Total ammonia } \\
\text { plus organic } \\
\text { nitrogen, } \\
\mathrm{mg} / \mathrm{L} \text { as } \mathrm{N}\end{array}$ & $\begin{array}{c}\text { Dissolved } \\
\text { ammonia plus } \\
\text { organic } \\
\text { nitrogen, } \\
\mathrm{mg} / \mathrm{L} \text { as } \mathrm{N}\end{array}$ & $\begin{array}{c}\text { Total } \\
\text { Phosphorus, } \\
\text { mg/L as } \mathrm{P}\end{array}$ & $\begin{array}{l}\text { Dissolved } \\
\text { Phosphorus, } \\
\text { mg/L as P }\end{array}$ & $\begin{array}{l}\text { Dissolved } \\
\text { orthophosphate } \\
\text { phosphorus, } \\
\text { mg/l as P }\end{array}$ \\
\hline Agnes & $<0.008$ & 0.07 & 0.06 & 1.5 & 0.6 & 0.025 & 0.009 & $<0.02$ \\
\hline Brown & $<0.008$ & 0.08 & 0.03 & 0.4 & 0.4 & 0.009 & 0.004 & $<0.02$ \\
\hline Cruiser $^{1}$ & -- & -- & -- & -- & -- & -- & -- & -- \\
\hline Ek & $<0.008$ & 0.13 & $<0.04$ & 1.3 & 0.5 & 0.012 & 0.006 & $<0.02$ \\
\hline Fishmouth & $<0.008$ & E0.03 & 0.26 & 0.7 & 0.7 & 0.008 & 0.004 & $<0.02$ \\
\hline Jorgens & $<0.008$ & 0.12 & E0.03 & 0.8 & 0.4 & 0.007 & 0.005 & $<0.02$ \\
\hline Little Shoepack & $<0.008$ & 0.17 & 0.05 & 1.5 & 0.5 & 0.012 & 0.005 & $<0.02$ \\
\hline Little Trout & $<0.008$ & $<0.05$ & $<0.04$ & 0.6 & 0.2 & 0.004 & $<0.004$ & $<0.02$ \\
\hline Locator & $<0.008$ & 0.07 & $<0.04$ & 1.1 & 0.3 & 0.01 & 0.004 & $<0.02$ \\
\hline Loiten & $<0.008$ & 0.09 & $<0.04$ & 0.9 & 0.3 & 0.006 & 0.004 & $<0.02$ \\
\hline Mukooda & $<0.008$ & $<0.05$ & $<0.04$ & 0.5 & 0.3 & 0.012 & 0.004 & $<0.02$ \\
\hline Net & $<0.008$ & 0.05 & $<0.04$ & 0.7 & 0.5 & 0.024 & 0.008 & $<0.02$ \\
\hline O’Leary & $<0.008$ & $<0.05$ & $<0.04$ & 1.0 & 0.3 & 0.014 & 0.005 & $<0.02$ \\
\hline Oslo & $<0.008$ & 0.08 & E0.02 & 0.5 & 0.4 & 0.012 & 0.005 & E0.01 \\
\hline Peary & $<0.008$ & 0.07 & $<0.04$ & 0.5 & 0.4 & 0.016 & 0.007 & $<0.02$ \\
\hline Quarter Line & $<0.008$ & 0.06 & $<0.04$ & 2.0 & 0.6 & 0.018 & 0.010 & $<0.02$ \\
\hline Quill & $<0.008$ & 0.12 & $<0.04$ & 0.8 & 0.3 & 0.007 & 0.004 & $<0.02$ \\
\hline Ryan & $<0.008$ & 0.06 & 0.13 & 0.6 & 0.6 & 0.009 & 0.005 & $<0.02$ \\
\hline Shoepack & $<0.008$ & 0.16 & E0.03 & 1.6 & 0.5 & 0.027 & 0.007 & $<0.02$ \\
\hline Tooth & $<0.008$ & E0.04 & $<0.04$ & 0.5 & 0.4 & 0.010 & 0.005 & $<0.02$ \\
\hline
\end{tabular}

${ }^{1}$ Cruiser Lake was not included in this study because of consistently low mercury and methylmercury concentrations, and because a collaborative fish study did not sample fish from this lake. 
Table 10. Major ion water chemistry in selected lakes, Voyageurs National Park, May 2002 $[\mathrm{mg} / \mathrm{L}$, milligrams per liter; $\mu \mathrm{g} / \mathrm{L}$, micrograms per liter; --not sampled; <, less than the method detection limit; E, estimated]

\begin{tabular}{|c|c|c|c|c|c|c|c|}
\hline Lake & $\begin{array}{l}\text { Total alkalinity, } \\
\mathrm{mg} / \mathrm{L} \text { as } \mathrm{CaCO}_{3}\end{array}$ & $\begin{array}{c}\text { Dissolved } \\
\text { sulfate, } \\
\mathrm{mg} / \mathrm{l} \text { as } \mathrm{SO}_{4}\end{array}$ & $\begin{array}{l}\text { Dissolved } \\
\text { chloride, } \\
\mathrm{mg} / \mathrm{L} \text { as } \mathrm{Cl}\end{array}$ & $\begin{array}{l}\text { Dissolved } \\
\text { fluoride, } \\
\mathrm{mg} / \mathrm{L} \text { as } \mathrm{F}\end{array}$ & $\begin{array}{c}\text { Dissolved } \\
\text { silica, } \\
\mathrm{mg} / \mathrm{L} \text { as } \mathrm{SiO}_{2}\end{array}$ & $\begin{array}{l}\text { Dissolved iron, } \\
\mu \mathrm{g} / \mathrm{L} \text { as } \mathrm{Fe}\end{array}$ & $\begin{array}{c}\text { Dissolved } \\
\text { manganese } \\
\mu \mathrm{g} / \mathrm{L} \text { as } \mathrm{Mn}\end{array}$ \\
\hline Agnes & 4 & 0.9 & 0.2 & $<0.1$ & 5.1 & 480 & 59 \\
\hline Brown & 6 & 1.5 & $<0.3$ & $<0.1$ & 2.8 & 160 & 3 \\
\hline Cruiser $^{1}$ & -- & -- & -- & -- & -- & -- & -- \\
\hline Ek & 12 & 1.1 & $<0.3$ & $<0.1$ & 2.0 & 250 & 2 \\
\hline Fishmouth & 12 & 1.4 & 0.2 & $<0.1$ & 0.77 & 26 & 2 \\
\hline Jorgens & 7 & 1.7 & $<0.3$ & $<0.1$ & 2.5 & 280 & 40 \\
\hline Little Shoepack & 9 & 1.5 & $<0.3$ & $<0.1$ & 2.2 & 160 & 3 \\
\hline Little Trout & 15 & 2.7 & 0.2 & E0.06 & 0.49 & $<10$ & $<2$ \\
\hline Locator & 6 & 2.3 & 0.4 & $<0.1$ & 2.8 & 190 & 6 \\
\hline Loiten & 7 & 2.6 & 0.3 & $<0.1$ & 2.6 & 130 & 4 \\
\hline Mukooda & 26 & 1.7 & 0.2 & $<0.1$ & 0.81 & 11 & $<2$ \\
\hline Net & 11 & 2.2 & 0.2 & $<0.1$ & 3.0 & 170 & 3 \\
\hline O'Leary & 31 & 1.9 & 0.2 & $<0.1$ & 0.87 & 25 & $<2$ \\
\hline Oslo & 6 & 1.4 & 0.2 & $<0.1$ & 3.6 & 190 & 4 \\
\hline Peary & 12 & 1.4 & 0.2 & $<0.1$ & 2.4 & 260 & 14 \\
\hline Quarter Line & 6 & 1.7 & 0.2 & $<0.1$ & 3.0 & 340 & 17 \\
\hline Quill & 7 & 2.3 & 0.4 & $<0.1$ & 2.5 & 190 & 6 \\
\hline Ryan & 8 & 3.0 & 0.3 & $<0.1$ & 2.9 & 220 & 39 \\
\hline Shoepack & 7 & 1.6 & 0.2 & $<0.1$ & 3.7 & 470 & 82 \\
\hline Tooth & 13 & 2.9 & 0.2 & E0.07 & 2.4 & 72 & 3 \\
\hline
\end{tabular}

${ }^{1}$ Cruiser Lake was not included in this study because of consistently low mercury and methylmercury concentrations, and because a collaborative fish study did not sample fish from this lake. 


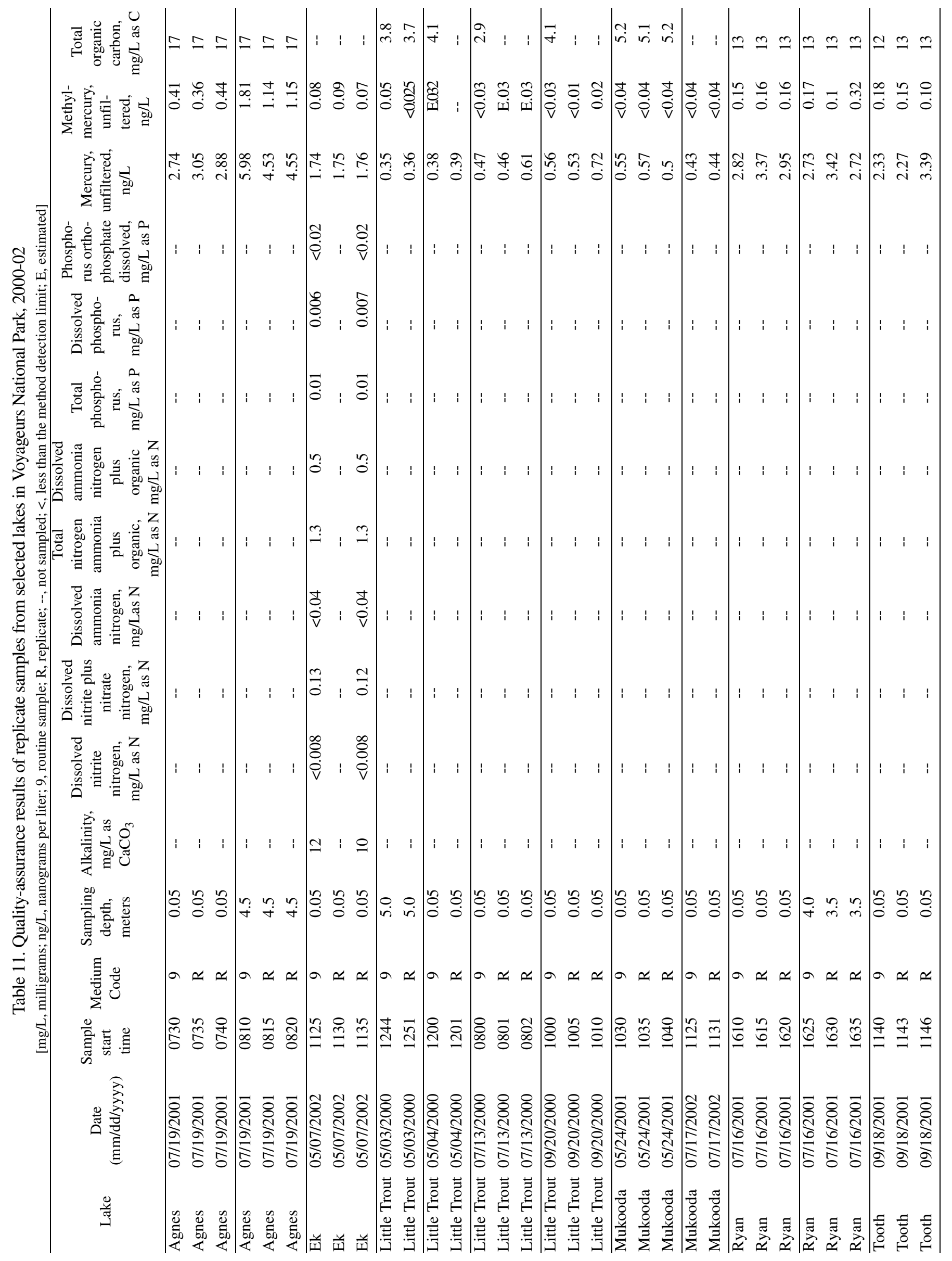


Table 12. Quality-assurance results of blanks from selected lakes, Voyageurs National Park, 2000-02

[mm/dd/yyyy, month, date, year; mg/L, milligrams per liter; ng/L, nanograms per liter; E estimated;--, not sampled; <, less than the method detection limit]

\begin{tabular}{lcccccc} 
[mm/dd/yyyy, month, date, year; mg/L, milligrams per liter; ng/L, nanograms per liter; E estimated;--, not sampled; <, less than the method detection limil \\
\hline \multicolumn{1}{c}{ Lake } & $\begin{array}{c}\text { Sample date } \\
(\mathrm{mm} / \mathrm{dd} / \text { yyyy) }\end{array}$ & $\begin{array}{c}\text { Sample start } \\
\text { time }\end{array}$ & Type of sample & $\begin{array}{c}\text { Mercury, } \\
\text { unfiltered, ng/L }\end{array}$ & $\begin{array}{c}\text { Methylmercury, } \\
\text { unfiltered, ng/L }\end{array}$ & $\begin{array}{c}\text { Total organic } \\
\text { carbon, } \\
\mathrm{mg} / \mathrm{L} \text { as C }\end{array}$ \\
\hline Fishmouth & $09 / 19 / 2001$ & 1200 & field blank & 0.79 & -- & -- \\
Little Shoepack & $05 / 23 / 2001$ & 1630 & field blank & $<0.04$ & $<0.04$ & $<0.04$ \\
Locator & $09 / 22 / 2000$ & 1000 & office blank & 0.11 & $<.03$ & 0.1 \\
Locator & $07 / 19 / 2001$ & 1235 & pump blank & 0.15 & $<0.04$ & 0.3 \\
Mukooda & $09 / 18 / 2001$ & 1105 & field blank & 0.09 & $<0.04$ & $<0.04$ \\
Mukooda & $07 / 17 / 2002$ & 1132 & field blank & 0.05 & $<0.04$ & -- \\
Shoepack & $0920 / 2001$ & 1240 & field blank & 0.13 & $<0.04$ & 0.14 \\
Tooth & $07 / 18 / 2001$ & 1300 & pump blank & 0.10 & $<0.04$ & -- \\
\hline
\end{tabular}




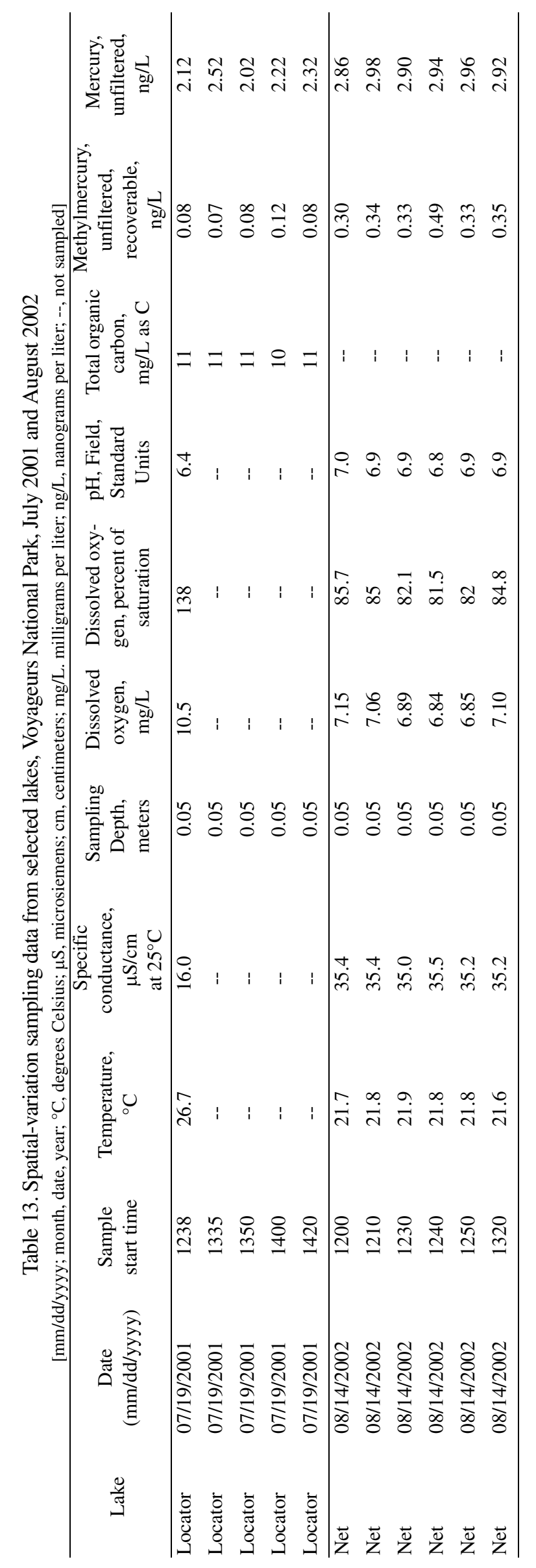




\section{REFERENCES}

American Public Health Association, American Water Works Association, and Water Environment Federation, 1998, Standard methods for the examination of water and wastewater (20th ed.): Baltimore, MD, United Book Press, Inc., variously paged.

Barr, J.F., 1986, Population dynamics of the common loon (Gavia immer) associated with mercury-contaminated waters in northwestern Ontario: Canadian Wildlife Service Canadian Wildlife Service Occasional Paper 56.

Brumbaugh, W.G., Krabbenhoft, D.P., Helsel, D.R., Weiner, J.G., and Echols, K.R., 2001, A national pilot study of mercury contamination of aquatic ecosystems along multiple gradients-Bioaccumulation in fish: U.S. Geological Survey USGS/BRD/BSR-P2001-0009, $25 \mathrm{p}$.

Clarkson, T.W., 1992, Mercury-Major issues in environmental health: Environmental Health Perspectives, v. 100, p. 31-38.

DeWild J.F., Olson, M.L., and Olund, S.D., 2002 Determination of methylmercury by aqueous phase ethylation, followed by gas chromatographic separation with cold vapor atomic fluorescence detection: U.S. Geological Survey Open-File Report 01-445, 14 p.

Engstrom, D.R., Thommes, K., Balogh, S.J., Swain, E.B., and Post, H.A., 1999, Trends in atmospheric mercury deposition across Minnesota-Evidence from dated sediment cores from 50 Minnesota lakes: St. Croix Watershed Research Station Draft Report to the Legislative Commission on Minnesota Resources, $32 \mathrm{p}$.

Friedmann, A.S., Watzin, M.C., BrinckJohnsen, T., and Leiter, J.C., 1996, Low levels of dietary methylmercury inhibit growth and gonadal development in juvenile walleye (Stizostedion vitreum): Aquatic Toxicology, v. 35, p. 265-278.

Kallemeyn, L.W., Holmberg, K.L., Perry, J.A., and Odde, B.Y., 2003,
Aquatic synthesis for Voyageurs National Park: Information and Technology Report USGS/BRD/ITR-2003-0001, 95 p.

Krabbenhoft, D.P., and Rickert, D.A., 1995, Mercury contamination of aquatic ecosystems: U.S. Geological Survey Open-File Report 95-216, $4 \mathrm{p}$.

Meyer, M.W., Evers, D.C., Daulton, T., and Braselton, W.E., 1995, Common loons (Gavia immer) nesting on low $\mathrm{pH}$ lakes in northern Wisconsin have elevated blood mercury content:

Water, Air, and Soil Pollution, v. 80, p. 871-880.

Meyer, M.W., Evers, D.C., Hartigan, J.J., and Rasmussen, P.S., 1998, Patterns of common loon (Gavia immer) mercury exposure, reproduction, and survival in Wisconsin, USA: Environmental Toxicology and Chemistry, v. 17, no. 2, p. 184-190.

Minnesota Department of Health, 1998, Minnesota fish consumption advisory: Minnesota Department of Health, $91 \mathrm{p}$.

Olson, M.L., and DeWild, J.F., 1999, Techniques for the collection and species-specific analysis of low levels of mercury in water, sediment, and biota, in Morganwalp, D.W., Buxton, H.T., eds., U.S. Geological Survey Toxic Substances Hydrology Program-Proceedings of the technical meeting, Charleston, South Carolina, March 8-12, 1999: U.S. Geological Survey Water-Resources Investigations Report 99-4018, p. 191-200.

Scheuhammer, A.M., 1991, Effects of acidification on the availability of toxic metals and calcium to wild birds and mammals: Environmental Pollution, v. 71, p. 329-375.

Scheuhammer, A.M., and Blancher, P.J., 1994, Potential risk to common loons (Gavia immer) from methylmercury exposure in acidified lakes: Hydrobiologia, v. 278/280, p. 445-455.

St. Louis, V.L., Rudd, J.W.M., Kelly, C.A., Beaty, K.G., Bloom, N.S., and Flett, R.J., 1994, Importance of wetlands as sources of methylmercury to boreal forest ecosystems: Canadian Journal of Fisheries and Aquatic Sci- ences, v. 51, p. 1065-1076.

Swain, E.B., Engstrom, D.R., Brigham, M.E., Henning, T.A., and Brezonik, P.L., 1992, Increasing rates of atmospheric mercury deposition in midcontinental North America: Science, v. 257, p. 784-787.

Swain, E.B., and Helwig, D.D., 1989, Mercury in fish from northeastern Minnesota lakes-Historical trends, environmental correlates, and potential sources: Journal of Minnesota Academy of Science, v. 55, no. 1, p. 103-109.

U.S. Environmental Protection Agency, 1996, Method 1669-Sampling ambient water for trace metals at EPA water quality criteria levels: U.S. Environmental Protection Agency EPA 821/R-96-011, 35 p.

U.S. Environmental Protection Agency, 1997, Mercury study report to Congress volume $\mathrm{V}-$ Health effects of mercury and mercury compounds: U.S. Environmental Protection Agency EPA-452/R-97-007, 349 p.

U.S. Environmental Protection Agency, 1999, Method 1631, revision - Mercury in water by oxidation, purge, and trap, and cold vapor atomic fluorescence spectrometry: U.S. Environmental Protection Agency EPA-821R-99-005, 33 p.

U.S. Environmental Protection Agency, 2001, Water quality criterion for the protection of human health-Methylmercury: Office of Science and Technology, Office of Water, USEPA EPA-823-R-01-001.

U.S. Geological Survey, 2003, Waterquality samples for Minnesota: U.S. Geological Survey, accessed October 28, 2003, at URL http://nwis.waterdata.usgs.gov/mn/nwis/qwdata

Wiener, J.G., and Spry, D.J., 1996, Toxicological significance of mercury in freshwater fish, in Beyer, W.N., Heinz, G.H., and Redmon, A.W., eds., Environmental Contaminants in Wildlife: Interpreting Tissue Concentrations: Boca Raton, FL, Lewis Publishers, p. 297-339. 